\title{
Collimated positronium production from gases ${ }^{\star}$
}

\author{
Michael Shipman ${ }^{1, a}$, Simon J. Brawley ${ }^{1}$, László Sarkadi² ${ }^{2}$ and Gaetana Laricchia ${ }^{1, b}$ \\ 1 UCL Department of Physics and Astronomy, University College London, Gower St., London, WC1E 6BT, UK \\ 2 Institute of Nuclear Research of the Hungarian Academy of Sciences (ATOMKI), 4001 Debrecen, P.O. Box 51, Hungary
}

Received 12 December 2013 / Received in final form 4 February 2014

Published online 3 April 2014

(c) The Author(s) 2014. This article is published with open access at Springerlink.com

\begin{abstract}
New data for positronium beam production efficiency are presented. As a converter of positrons to forward-going positronium, $\mathrm{O}_{2}$ has been found to be as good as $\mathrm{CO}_{2}$ from $\sim 250$ to $400 \mathrm{eV}$. Preliminary data is also presented for the production efficiency from $\mathrm{Ar}$ at $2.8 \mathrm{eV}$.
\end{abstract}

\section{Introduction}

Positronium (Ps) is the lightest known atom. A matterantimatter hybrid, it may be formed in one of two spin states: the singlet ${ }^{1} \mathrm{~S}_{0}$ has a ground-state lifetime against annihilation, $\tau_{\mathrm{Ps}}$, of $125 \mathrm{ps}$, and the triplet, ${ }^{3} \mathrm{~S}_{1}$, of $142 \mathrm{~ns}$. It is a hydrogenic atom whose formation probability by positrons colliding with matter is considerable, accounting in atoms for $\sim 50 \%$ at its peak [1]. In fact, about $93 \%$ of all $\gamma$-rays observed from the galactic centre and over $80 \%$ of the $\gamma$-rays emitted in PET scans are estimated to originate from the annihilation of the Ps atom $[2,3]$. Ps is also a good model system for tests of QED theory given the lack of complications from hadronic structures, and investigations into Ps-matter processes are an aid to understanding basic scattering interactions and the development of accurate scattering theories [4].

In collisions of Ps with matter, the first-order polarization and static interactions are zero, due respectively to its neutrality and coincidence of its centres of mass and charge. Thus, in comparison to an electron for which both interactions are non-zero, exchange is expected to play a comparatively larger part [5]. Yet Brawley et al. [6] observed that for all targets and energies so far investigated (i.e. $\mathrm{He}, \mathrm{Ne}, \mathrm{Ar}, \mathrm{Kr}, \mathrm{Xe}, \mathrm{H}_{2}, \mathrm{~N}_{2}, \mathrm{O}_{2}, \mathrm{SF}_{6}$ and $\mathrm{CO}_{2}[6,7]$ between 7 and $400 \mathrm{eV}$ ), the Ps atom has a similar total cross section, $Q_{\mathrm{T}}^{\mathrm{Ps}}$, to that of an equivelocity electron, $Q_{\mathrm{T}}^{-}$, even near velocities at which resonances are observed in $Q_{\mathrm{T}}^{-}$as in $\mathrm{CO}_{2}$ [7].

\footnotetext{
* Contribution to the Topical Issue "Electron and Positron Induced Processes", edited by Michael Brunger, Radu Campeanu, Masamitsu Hoshino, Oddur Ingólfsson, Paulo Limão-Vieira, Nigel Mason, Yasuyuki Nagashima and Hajime Tanuma.

a e-mail: michael.shipman.09@ucl.ac.uk

b e-mail: g.laricchia@ucl.ac.uk
}

Neutral Ps may be formed by implanting positrons into insulators (e.g. [8]), or upon positron re-emission from metals $[9,10]$, or by scattering high energy positrons at glancing angles from metal surfaces [11]. In the last case, fast Ps atoms typically have energy spreads inadequate for measurements at specific energies, and ill-defined quantum states [12]. Ps formed by passing a positron beam through a suitable gas, however, has been found to have the advantages of dominant ground state production, and energies (and associated widths) directly related to those of incoming beam, e.g. [13,14]. A novel method for producing a Ps beam, energy-tunable over the range $300 \mathrm{eV}$ to $1.9 \mathrm{keV}$, has also been achieved by Michishio et al. in which the beam is formed from the photoionisation of $\mathrm{Ps}^{-}$[15].

At UCL, the Ps beam is produced using the positrongas technique (see Sects. 2 and 3). Over the years, the efficiency of this conversion has been investigated for various targets as a function of energy and target pressure, e.g. $[16,17]$. Below $\sim 100 \mathrm{eV}, \mathrm{H}_{2}$ has been found to be the most efficient production target. More recently, motivated by the results of Cooke et al. [18] who found that the integrated Ps formation cross section, $Q_{\mathrm{Ps}}$, for both $\mathrm{CO}_{2}$ and $\mathrm{N}_{2}$ remained significant up to about $800 \mathrm{eV}$, Shipman et al. [17] investigated $\mathrm{N}_{2}$ and $\mathrm{CO}_{2}$ as a production gas up to $E_{\mathrm{Ps}} \sim 400 \mathrm{eV} . \mathrm{CO}_{2}$ was found to be the best converter above $250 \mathrm{eV}$ and twice as efficient as the next best, $\mathrm{N}_{2}$, at these energies. The present work extends this type of investigation to $\mathrm{O}_{2}$.

The Ps beam energy resolution and low production efficiencies have thus far been a limiting factor in the investigation of the rich resonance structure occurring at low energies in $Q_{\mathrm{T}}^{-}$for many molecules [19]. For this reason, we have also investigated Ar with respect to its beam production efficiency at low velocities where a comparison with electron cross-sections suggests that $Q_{\mathrm{T}}^{\mathrm{Ps}}$ (and thus the attenuation of the Ps beam out of the production cell) might be small. 


\section{Experimental apparatus}

The beam line at UCL has been described fully elsewhere, e.g. [14]. In brief, energetic $\beta^{+}$particles produced by a ${ }^{22} \mathrm{Na}$ source are moderated by a rare gas solid - of Ar, $\mathrm{Kr}$ or $\mathrm{Ne}$ - frozen onto the source. A positive potential with respect to the chamber earth is applied to the source and accelerates the positrons. The beam is confined and guided by a magnetic field of $\sim 10^{-2} \mathrm{~T}$ produced by eleven Helmholtz coils along a flight path of $\sim 2 \mathrm{~m}$. The positrons are deflected by a Wien filter (such that no line of sight exists between the source and the detectors) and guided into a Ps production gas cell. Ps atoms are formed in the charge-exchange reaction $\mathrm{e}^{+}+\mathrm{A} \longrightarrow \mathrm{Ps}+\mathrm{A}^{+}$, where $\mathrm{A}$ is the production target, and those that travel in the forward direction may enter a second cell in which a target gas can be contained. Transmitted positrons are repelled by a positive potential applied to a $\mathrm{W}$ grid between the cells. The Ps beam is detected by impacts on a CEM or CEMA monitored in coincidence with correlated annihilation signals from three nearby $\gamma$-ray detectors. From time-of-flight measurements, the energy of the Ps beam, $E_{\mathrm{Ps}}$, has been found to obey the relationship: $E_{\mathrm{Ps}}=E_{+}-E_{\mathrm{I}}+6.8 / n^{2} \mathrm{eV}$, where $E_{+}, E_{\mathrm{I}}$ and $n$ are the positron beam energy, the first ionisation energy of the production target and the Ps principal quantum number, respectively. Time-of-flight studies have also determined that the Ps beam is formed predominantly in the ground state with an energy spread approximately that of the positron beam, i.e. $\sim 2 \mathrm{eV}$ FWHM [20].

\section{Results}

The production efficiency, $\epsilon_{\mathrm{Ps}}$, for a beam of Ps atoms within a small angle $\Omega$ (the solid angle subtended by the detector with respect to the production point of the Ps beam) is a function of $E_{\mathrm{Ps}}$ and pressure, $P$, and defined as:

$$
\epsilon_{\mathrm{Ps}}\left(E_{\mathrm{Ps}}, P\right)=\frac{N_{\mathrm{Ps}}}{N_{+}} \frac{1}{\Omega} \frac{1}{S} \frac{\eta_{+}}{\eta_{\mathrm{Ps}}},
$$

where $N_{\mathrm{Ps}}$ corresponds to the Ps counts, $N_{+}$to that for positrons entering the production cell, $S=\exp \left(-t / \tau_{\mathrm{Ps}}\right)$ is the Ps fraction that reaches the detector in time $t$, and $\eta_{\mathrm{Ps}}$ and $\eta_{+}$are the detection efficiencies for Ps atoms and positrons, respectively, the ratio $\eta_{+} / \eta_{\mathrm{Ps}}$ having been found to be approximately constant across different detectors and over long periods of time [17]. In terms of the total cross sections for positrons and $\operatorname{Ps}\left(Q_{\mathrm{T}}^{+}\right.$and $Q_{\mathrm{T}}^{\mathrm{Ps}}$, respectively), the production efficiency may be expressed as:

$$
\begin{array}{r}
\epsilon_{\mathrm{Ps}} \propto\left\{1-\exp \left(-\rho l_{+} Q_{\mathrm{T}}^{+}\right)\right\}\left\{\frac{1}{Q_{\mathrm{T}}^{+}} \int_{0}^{\theta^{\prime}} \frac{d Q_{\mathrm{Ps}}}{d \Omega} \sin \theta d \theta\right\} \\
\times \exp \left(-\rho l_{\mathrm{Ps}} Q_{\mathrm{T}}^{\mathrm{Ps}}\right),
\end{array}
$$

where $\rho$ is the number density of the target gas, and $l_{+}$ and $l_{\mathrm{Ps}}$ are the effective cell lengths for positrons and for positronium, respectively. The first term of equation (2) is the fraction of positrons scattered, the second term the probability of forming Ps within the angular range 0 to $\theta^{\prime}$, and the third term the transmission probability of the Ps through the cell.

The production efficiency from $\mathrm{O}_{2}$ was investigated at twelve Ps energies between 10.5 and $399 \mathrm{eV}$. Figure 1 shows the present results for $\mathrm{O}_{2}$ as a function of pressure in comparison to those for $\mathrm{H}_{2}[13,17,21], \mathrm{N}_{2}[13,17,21]$, Ar [13] and $\mathrm{CO}_{2}$ [17] ( $\epsilon_{\mathrm{Ps}}$ for each data set has been corrected for the detector solid angle and in-flight annihilation as in Eq. (1), but uncorrected for the final term, $\left.\frac{\eta_{+}}{\eta_{\mathrm{Ps}}}\right)$. In general, for each production target, $\epsilon_{\mathrm{Ps}}$ shows a rise to a plateau, in some cases decreasing with increasing pressure in the range measured due to attenuation of the Ps beam itself. $\epsilon_{\mathrm{Ps}}$ is highly dependent on the target and $E_{\mathrm{Ps}}$, and its maximum values found as a function of pressure for a given $E_{\mathrm{Ps}}$, are shown for $\mathrm{O}_{2}$ in Figure 2 where they are compared to those for $\mathrm{H}_{2}, \mathrm{CO}_{2}, \mathrm{~N}_{2}$ and $\mathrm{Xe}$ from $\sim 10$ to $400 \mathrm{eV}$.

As generally found for other targets, $\epsilon_{\mathrm{Ps}}$ for $\mathrm{O}_{2}$ increases rapidly from low energies, as the Ps formation cross-section rises and becomes more forward-peaked [22]. A broad maximum is observed around 40 to $60 \mathrm{eV}$, followed by a gradual decrease to higher energies. Below $\sim 100 \mathrm{eV}, \mathrm{O}_{2}$ is the second poorest production target after Xe, but falls less rapidly than $\mathrm{H}_{2}$ and $\mathrm{N}_{2}$ with increasing energy and exceeds the latter by $80 \%$ at $250 \mathrm{eV}$. From $250 \mathrm{eV}$ upwards, $\epsilon_{\mathrm{Ps}}$ is the same as for $\mathrm{CO}_{2}, 2.5$ times that for $\mathrm{N}_{2}$ by $400 \mathrm{eV}$, though two orders of magnitude lower than the peak for the most efficient converter, $\mathrm{H}_{2}$.

At the lowest energy investigated, $\epsilon_{\mathrm{Ps}}$ for $\mathrm{O}_{2}$ is found to be only $6 \%$ that of $\mathrm{H}_{2}$. It may be of interest to note that the positron total ionisation cross section for $\mathrm{O}_{2}$ at $10 \mathrm{eV}$ - where Ps formation is generally expected to dominate over annihilation [23] - experiences a local minimum at the same energy as channels open for positron impact excitation to the Schumann-Runge continuum [24]. Low Ps beam production may also be the result of $\mathrm{Ps}^{3} \mathrm{~S}_{1}$ quenching to the short-lived ${ }^{1} \mathrm{~S}_{0}$ state (e.g. $[25,26])$. Below $1 \mathrm{eV}$, this conversion has been found to proceed elastically and inelastically via excitations to the $\mathrm{a}^{1} \Delta_{\mathrm{g}}$ and $\mathrm{b}^{1} \Sigma_{\mathrm{g}}^{+}$states $[27,28]$, and whilst the former has a small cross section $\left(\sim 10^{-23} \mathrm{~m}^{2}\right)$, the inelastic contribution has been found to be two orders of magnitude greater. Measurements around $10 \mathrm{eV}$ Ps impact energy are not currently available, but cross sections for $\mathrm{X}^{3} \Sigma_{\mathrm{g}}^{-} \rightarrow$ $\mathrm{a}^{1} \Delta_{\mathrm{g}}$ and $\mathrm{X}^{3} \Sigma_{\mathrm{g}}^{-} \rightarrow \mathrm{b}^{1} \Sigma_{\mathrm{g}}^{+}$excitations have been measured at these energies for electron collisions by Trajmar et al. [29], Middleton et al. [30], Shyn and Sweeny [31] and Linert and Zubek [32] who report cross sections around $10^{-21} \mathrm{~m}^{2}$. Whilst this only contributes about $1 \%$ to $Q_{\mathrm{T}}^{-}$, the $\mathrm{X}^{3} \Sigma_{\mathrm{g}}^{-} \rightarrow \mathrm{a}^{1} \Delta_{\mathrm{g}}$ and $\mathrm{X}^{3} \Sigma_{\mathrm{g}}^{-} \rightarrow \mathrm{b}^{1} \Sigma_{\mathrm{g}}^{+}$excitations proceed predominantly via the exchange interaction, and may thus be enhanced for Ps impact as discussed above. Further work is planned to carefully investigate $\epsilon_{\mathrm{Ps}}$ and $Q_{\mathrm{T}}^{\mathrm{Ps}}$ over these energies.

Preliminary measurements of the Ps beam production efficiencies from $\mathrm{Ar}$ at $E_{\mathrm{Ps}}=2.8 \mathrm{eV}$ are presented in Figure 3 . Whilst the precision is currently poor, there is a 

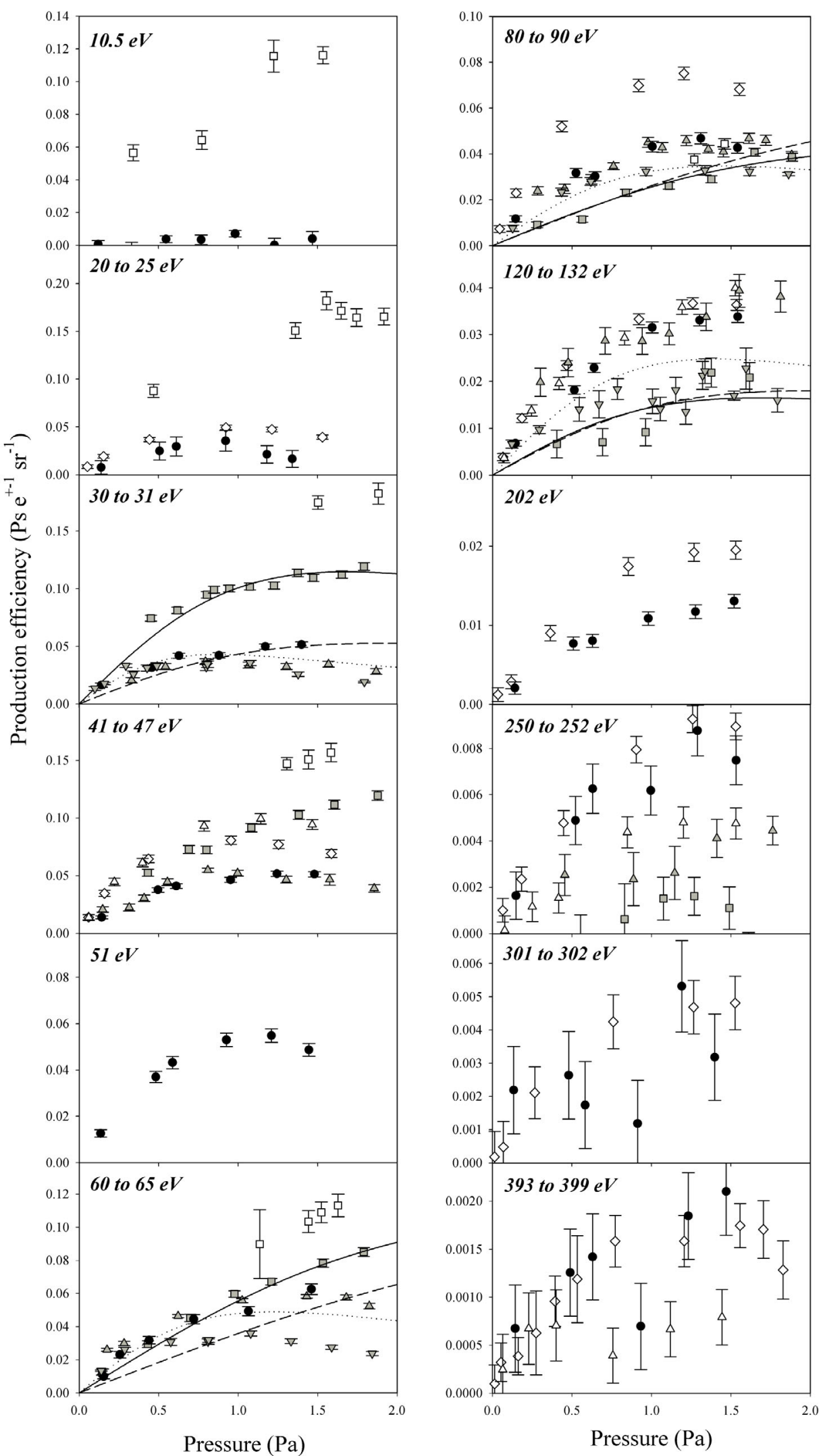

Fig. 1. The Ps production efficiency for $\mathrm{O}_{2}$ at selected energies as a function of pressure in comparison with other production targets. Circles: $\mathrm{O}_{2}$; squares: $\mathrm{H}_{2}$; up triangles: $\mathrm{N}_{2}$; diamonds: $\mathrm{CO}_{2}$; down triangles: Xe. Black points: this study; white points: Shipman et al. [17]; grey points: Leslie [21]. Lines are the measurements of Garner et al. [13]: $\mathrm{H}_{2} ;--\cdots: \mathrm{He} ; \cdots \cdots \cdots \cdot$ Ar. 


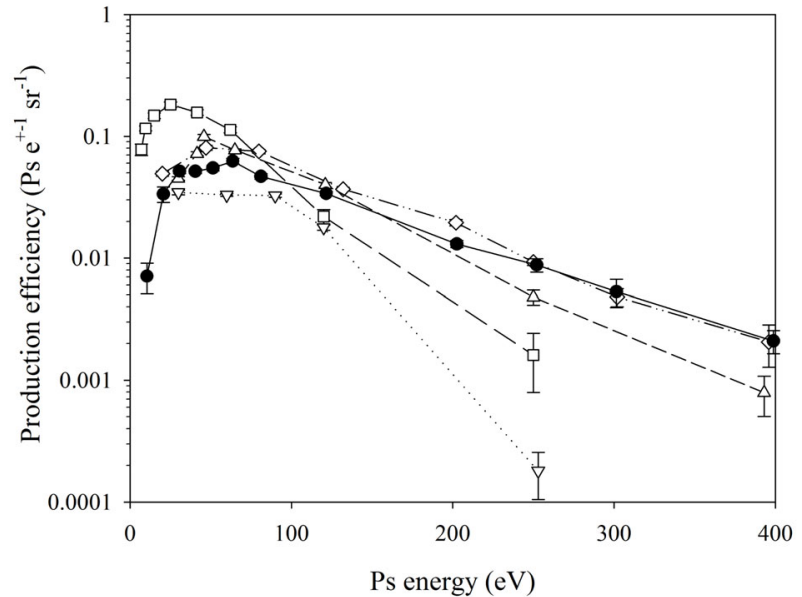

Fig. 2. Maximum Ps production efficiencies for various production targets. $\bullet, \mathrm{O}_{2}$ : this work; $\square: \mathrm{H}_{2}[17,21] ; \diamond: \mathrm{CO}_{2}$ [17]; $\triangle: \mathrm{N}_{2}[17,21] ; \nabla: \mathrm{Xe}[21]$.

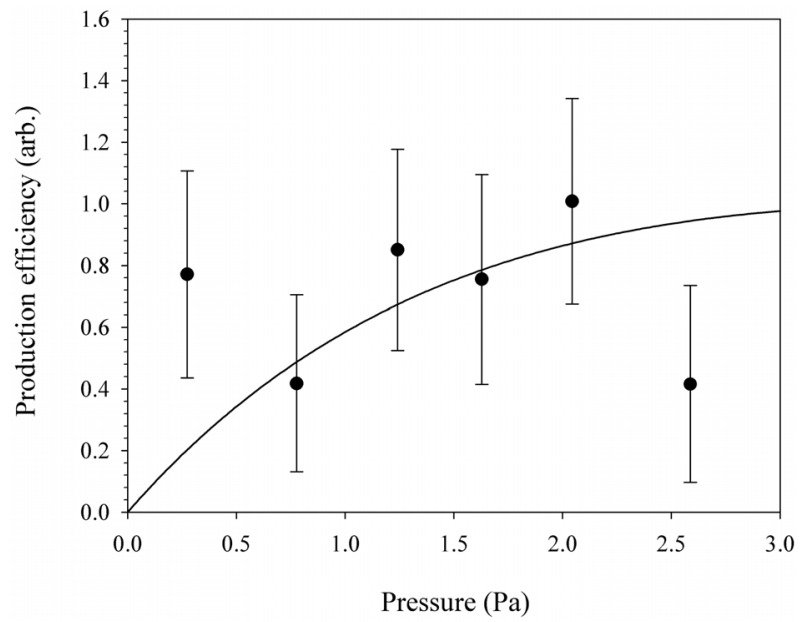

Fig. 3. Ps beam production efficiency at $E_{\mathrm{Ps}}=2.8 \mathrm{eV}$ (given in arbitrary units) for $\mathrm{Ar}$ as a function of pressure. The line displays the pressure dependence of equation (2) with $E_{+}=11.8 \mathrm{eV}, Q_{\mathrm{T}}^{\mathrm{Ps}}=Q_{\mathrm{T}}^{-}$at the same projectile velocity; $l_{+}=3.72 \mathrm{~cm}$ and $l_{\mathrm{Ps}}=l_{+} / 2$.

non-zero signal which, as illustrated in the figure, is not inconsistent with the pressure dependence of $\epsilon_{\mathrm{Ps}}$ computed from equation (2) with $Q_{\mathrm{T}}^{+} \simeq 5.48 \times 10^{-20}$ at $11.8 \mathrm{eV}$ [33], and $Q_{\mathrm{T}}^{\mathrm{Ps}} \simeq Q_{\mathrm{T}}^{-}$at the same projectile velocity [34], in accordance with the previously observed electron-like scattering of Ps $[6]$; the values for $l_{+}$and $l_{\mathrm{Ps}}$ are given in the caption. Further work is planned for low-energy production from this target.

\section{Conclusions and outlook}

We have extended to $\mathrm{O}_{2}$ the investigation of the efficiency of production of collimated Ps and found it to be as good as $\mathrm{CO}_{2}$ between 250 and $400 \mathrm{eV}$. We have also presented preliminary measurements of $\epsilon_{\mathrm{Ps}}$ for $\mathrm{Ar}$ at the Ps energy $E_{\mathrm{Ps}}=2.8 \mathrm{eV}$. Whilst much work remains to be carried out, the present results are not inconsistent with expectations, assuming $Q_{\mathrm{T}}^{\mathrm{Ps}} \approx Q_{\mathrm{T}}^{-}$at the same projectile velocity which is close to the well-known Ramsauer-Townsend minimum observed for electrons in collision with this target. Detailed energy profiles for the Ps beam produced from $\mathrm{O}_{2}, \mathrm{CO}_{2}$ and $\mathrm{Ar}$ will be investigated further, as will the Ps beam production efficiencies and total cross sections from $\mathrm{O}_{2}$ over the range of energies near $10 \mathrm{eV}$ at which a minimum is found in the total ionisation cross section for positron impact [24].

The authors kindly thank John Dumper and Rafid Jawad for their technical expertise, and EPSRC for funding this research under grant EP/J003980/1.

\section{References}

1. G. Laricchia, S. Armitage, A. Kövér, D.J. Murtagh, Adv. At. Mol. Opt. Phys. 56, 1 (2008)

2. J.C. Higdon, R.E. Lingenfelter, R.E. Rothschild, ApJ 698, $350(2009)$

3. C. Champion, C. Le Loirec, Phys. Med. Biol. 52, 6605 (2007)

4. G. Laricchia, H.R.J. Walters, Riv. Nuovo Cimento 35, 305 (2012)

5. J.E. Blackwood, C.P. Campbell, M.T. McAlinden, H.R.J. Walters, Phys. Rev. A 60, 4454 (1999)

6. S.J. Brawley, S. Armitage, J.E. Beale, D.E. Leslie, A. Williams, G. Laricchia, Science 330, 789 (2010)

7. S.J. Brawley, A.I. Williams, M. Shipman, G. Laricchia, Phys. Rev. Lett. 105, 263401 (2010)

8. D.B. Cassidy, A.P. Mills, Nature 449, 195 (2007)

9. A.P. Mills, W.S. Crane, Phys. Rev. A 31, 593 (1985)

10. R.H. Howell, I.J. Rosenberg, M.J. Fluss, Phys. Rev. B 34, 3069 (1986)

11. D.W. Gidley, R. Mayer, W.E. Frieze, K.G. Lynn, Phys. Rev. Lett. 58, 595 (1987)

12. G. Laricchia, in Positron Spectroscopy of Solids, Proceedings of the International School of Physics "Enrico Fermi", Course $C X X V$, edited by A. Dupasquier, A.P. Mills (1995), Vol. 125, pp. 401-418

13. A.J. Garner, G. Laricchia, A. Ozen, J. Phys. B 29, 5961 (1996)

14. A. Özen, A.J. Garner, G. Laricchia, Nucl. Instrum. Methods Phys. Res. B 171, 172 (2000)

15. K. Michishio, T. Tachibana, R.H. Suzuki, K. Wada, A. Yagishita, T. Hyodo, Y. Nagashima, Appl. Phys. Lett. 100, 254102 (2012)

16. D.E. Leslie, S. Armitage, G. Laricchia, J. Phys. B 35, 4819 (2002)

17. M. Shipman, S. Brawley, D.E. Leslie, S. Armitage, G. Laricchia, Eur. Phys. J. D 66, 96 (2012)

18. D.A. Cooke, D.J. Murtagh, G. Laricchia, J. Phys.: Conf. Ser. 199, 012006 (2010)

19. G.P. Karwasz, R.S. Brusa, A. Zecca, Riv. Nuovo Cimento 24, 1 (2001)

20. G. Laricchia, S. Armitage, D.E. Leslie, Nucl. Instrum. Methods Phys. Res. B 221, 60 (2004)

21. D.E. Leslie, Ph.D. thesis, UCL, 2005

22. C.J. Brown, J.W. Humberston, J. Phys. B 18, L401 (1985) 
23. P. Van Reeth, G. Laricchia, J.W. Humberston, Phys. Scr. 71, C9 (2005)

24. G. Laricchia, J. Moxom, M. Charlton, Phys. Rev. Lett. 70, 3229 (1993)

25. R. Ferrell, Phys. Rev. 110, 1355 (1958)

26. N. Shinohara, N. Suzuki, T. Chang, T. Hyodo, Phys. Rev. A 64, 042702 (2001)

27. M. Kakimoto, T. Hyodo, T. Chiba, T. Akahane, T.B. Chang, J. Phys. B 20, L107 (1987)

28. M. Kakimoto, T. Hyodo, T.B. Chang, J. Phys. B 23, 589 (1990)

29. S. Trajmar, D.C. Cartwright, W. Williams, Phys. Rev. A 4, 1482 (1971)

30. A.G. Middleton, P.J.O. Teubner, M.J. Brunger, Phys. Rev. Lett. 69, 2495 (1992)
31. T.W. Shyn, C.J. Sweeny, Phys. Rev. A 47, 1006 (1993)

32. I. Linert, M. Zubek, Chem. Phys. Lett. 492, 395 (2006)

33. A. Zecca, L. Chiari, E. Trainotti, D.V. Fursa, I. Bray, A. Sarkar, S. Chattopadhyay, K. Ratnavelu, M.J. Brunger, J. Phys. B 45, 015203 (2012)

34. J. Ferch, B. Granitza, C. Masche, W. Raith, J. Phys. B 18, $967(1985)$

Open Access This is an open access article distributed under the terms of the Creative Commons Attribution License (http://creativecommons.org/licenses/by/4.0), which permits unrestricted use, distribution, and reproduction in any medium, provided the original work is properly cited. 